\title{
Age, margin status, high-risk human papillomavirus and cytology independently predict recurrent high-grade cervical intraepithelial neoplasia up to 6 years after treatment
}

\author{
SONIA ANDERSSON ${ }^{1 *}$, DAVID MEGYESSI ${ }^{1 *}$, KAREN BELKIĆ $^{2-4}$, \\ SUSANNA ALDER $^{1}$, ELLINOR ÖSTENSSON ${ }^{1}$ and MIRIAM MINTS ${ }^{1,5}$
}

\begin{abstract}
${ }^{1}$ Department of Women's and Children's Health, Obstetrics-Gynecology Division; ${ }^{2}$ Department of Oncology-Pathology, Karolinska Institute, SE-17176 Stockholm, Sweden; ${ }^{3}$ School of Community/Global Health, Claremont Graduate University, Claremont, CA 91711; ${ }^{4}$ Institute for Health Promotion \& Disease Prevention Research, Keck School of Medicine, University of Southern California, Los Angeles, CA 90032, USA; ${ }^{5}$ School of Medical Sciences, Faculty of Medicine-Health, Örebrö University, SE-70182 Örebrö, Sweden
\end{abstract}

Received May 30, 2021; Accepted June 24, 2021

DOI: 10.3892/ol.2021.12945

\begin{abstract}
The present study aimed to identify the factors that independently contribute to disease recurrence among women first-time treated for high-grade cervical intraepithelial neoplasia (CIN) during 4-6 years of follow-up. Overall, 529 of 530 eligible patients participated; these patients all attended a 1st follow-up appointment $\sim 6$ months post-conization, at which time high-risk human-papillomavirus (HPV) testing, liquid-based cytology and colposcopy were performed. Full data on margin excision status, other aspects of initial treatment and comorbidity were obtained. At least one subsequent follow-up was attended by $88 \%$ of patients. A total of 22 recurrent cases were detected during follow-up. Detected recurrence was the outcome of focus for multiple logistic
\end{abstract}

Correspondence to: Professor Sonia Andersson, Department of Women's and Children's Health, Obstetrics-Gynecology Division, Karolinska Institute, Widerströmska Building, Tomtebodavägen 18A, SE-17176 Stockholm, Sweden

E-mail: sonia.andersson@ki.se

${ }^{*}$ Contributed equally

Abbreviations: AGC, atypical glandular cells; AIS, adenocarcinoma in-situ; ASC-H, atypical squamous cells cannot exclude HSIL; ASC-US, atypical squamous cells of undetermined significance; $\mathrm{CI}$, confidence intervals; CIN, cervical intraepithelial neoplasia; C-LETZ, contoured-loop excision of the transformation zone; HPV, high-risk human papillomavirus; HSIL, high-grade squamous intraepithelial lesions; LBC, liquid-based cytology; LSIL, low-grade squamous intraepithelial lesions; NILM, negative for intraepithelial lesions or malignancy; OR, odds ratio; PCR, polymerase chain reaction; TZ3, transformation zone 3; VSS, vaginal self-sampling

Key words: cervical intraepithelial neoplasia, treatment failure, margin status, papillomavirus infection, age regression analysis, with odds ratios (OR) and 95\% confidence intervals (CI) computed. Four significant independent risk factors were identified: Age 45 years or above $(\mathrm{OR}=3.5$, 95\% CI=1.3-9.9), one or both unclear or uncertain margins $(\mathrm{OR}=5.3,95 \% \mathrm{CI}=2.0-14.2)$, positive HPV at 1 st follow-up $(\mathrm{OR}=5.8,95 \% \mathrm{CI}=2.0-16.8)$, and abnormal cytology at $1 \mathrm{st}$ follow-up $(\mathrm{OR}=3.9,95 \% \mathrm{CI}=1.4-11.0)$. Bivariate analysis revealed that persistent HPV positivity was associated with recurrence $(\mathrm{P}<0.01)$. These findings indicated that incomplete excision of the CIN lesion may warrant more intensive subsequent screening, regardless of early post-conization HPV findings. Although early post-conization positive HPV was a powerful, independent predictor of recurrent high-grade CIN, over one-third of the patients with detected recurrence had a negative early post-conization HPV finding. These patients returned for routine screening, at which time, in most cases, HPV status was positive, thus indicating the need for repeated HPV evaluation. Especially during the on-going pandemic, home vaginal self-sampling is recommended. Particular attention is required for women aged $\geq 45$ years. In addition, although not statistically significant, relevant comorbidities, especially autoimmune conditions, warrant consideration in clinical decision-making. Women who have been treated for high-grade CIN are at risk for recurrent disease and progression to cervical cancer; therefore, they require careful, individualized follow-up to avoid these adverse consequences.

\section{Introduction}

Worldwide, in 2018 there were an estimated 570,000 cases of cervical cancer and 311,000 women died due to this malignancy (1). In Sweden, since the 1960s, an organized, population-based cervical screening program has substantially reduced cervical cancer incidence and mortality $(2,3)$. However, more recent data suggest that cervical cancer is on the rise once again in Sweden (4). A key aim of cervical screening programs, such as that in Sweden, is to identify and treat women with precancerous lesions, cervical 
intraepithelial neoplasia (CIN), before these lesions develop into invasive cancer (5). Once high-grade CIN has been identified and treated, more intense, prolonged follow-up is needed compared to the general population of women (6). Follow-up is of critical importance, because these patients are at long-term risk for developing recurrent disease $(7,8)$. Yet, evidence-based recommendations for the most appropriate post-therapeutic screening protocols are lacking (9).

It is now clearly-established that the high-risk human papilloma virus (HPV) is the key contributor to the development of cervical cancer. Consequently, testing for HPV is a vital component of all aspects of cervical cancer screening. This includes the use of HPV tests to evaluate the risk of recurrent disease in patients who have been treated for high-grade CIN (10). Numerous studies have shown that HPV testing provides essential information concerning the chances of disease recurrence in this vulnerable population of women (11-15).

A particular concern vis-à-vis cervical cancer risk is among women treated for high-grade CIN at a more advanced age. In the Swedish cancer registry study of all 132,493 women treated for CIN3 between 1958-2002, the relative risk of subsequent cervical cancer rose steadily with each decade of life. An accelerated risk was noted for women above 60 years of age (8). On the one hand, among women in the more senior age group, the overall prevalence of HPV positivity appears to be quite low (16). On the other hand, however, once infected with HPV, the infection may be more persistent than among younger women, and is associated with a high prevalence of cervical dysplasia (16-18). The potential contribution of persistent HPV infection to cervical cancer risk has, thus, been particularly emphasized for this age group. Further, these findings underscore the need to find appropriate algorithms for the more senior women to optimize cervical cancer surveillance (16).

Incomplete excision of high-grade CIN has also been implicated as a risk factor for recurrence of high-grade cervical dysplasia (19-23). In the literature, reports about the percentage of unclear or uncertain margins range widely, from as low as $3 \%$ up to as high as $50-60 \%$, with the overall percentage being approximately $23 \%$, according to a fairly-recent meta-analysis (20). Compared to when both the endocervical and ectocervical margins were considered clear, the risk was an estimated four to six-fold higher for recurrent high-grade cervical dysplasia with one or both unclear margins $(20,24)$.

Given the recognized etiologic role of HPV, however, questions have been raised about the actual importance of margin status in predicting recurrence after treatment of high-grade cervical dysplasia. In our 2020 long-term follow-up registry study of 991 women with histopathologically-confirmed high-grade CIN who underwent conization from 2000 to 2007 , a single post-conization HPV result was available for a small subgroup of the patients (19). Among the 84 patients with positive HPV findings, those with positive/uncertain margins showed a significantly increased risk of recurrent/residual CIN2+ (over two-fold) compared to women with HPV positive findings but with clear margins. In contrast, however, among the 105 women with negative post-conization HPV findings, margin status was not found to be significantly associated with recurrence. We noted the need for further investigation of this question with more complete post-conization HPV data for a cohort of patients followed after treatment. This is one of the aims of the present study.

In that light, most recently, within the framework of an investigation comparing clinician-sampled and self-sampled specimens at early post-conization follow-up, complete HPV and cytologic results, as well as practically complete colposcopic results were obtained for a large cohort of patients (25). We now incorporate these early post-conization data into a longer-term follow-up study extending for over six years. Full data on margin excision status and other aspects of initial treatment are included, as well as consideration of age and comorbid conditions that may impact on HPV acquisition and/or CIN progression $(19,26)$. Herein, our main focus is upon recurrence of high-grade cervical dysplasia, seeking to identify the factors that independently contribute to treatment failure.

\section{Materials and methods}

Design of the study, population and location. Between October 2014 and January 2017, all patients who had been treated for the first time by conization for histologically-confirmed high-grade CIN (CIN2+) or adenocarcinoma in-situ (AIS) were eligible to participate in this study. The hospitals in which the patients had been treated were: Karolinska University Hospital, Danderyd Hospital or South General Hospital, all within Stockholm County, Sweden.

The patients were contacted soon after treatment by Ellinor Östensson, EÖ, who scheduled the 1st follow-up visit at Karolinska University Hospital. Approximately six months post-conization was the aimed time interval for this 1st follow-up visit. Often after repeated attempts to find a suitable time, all 532 patients were scheduled and then attended this 1st follow-up.

After coming to the Karolinska University Hospital for the 1st follow-up visit, EÖ met with each woman to present the study procedures. These included self-collection of vaginal and urine samples for HPV testing, as reported in a previous study (25); completion of a questionnaire, as reported in previous studies $(27,28)$; gynecologic examination with colposcopy and cervical sampling as clinical follow-up. The overall aim of the study was stated to be prevention of cervical cancer. The participants were assured of complete confidentiality and full freedom to withdraw from the study at any time with no consequences whatsoever. Permission to review the patient's medical records was included in the informed consent. The options for the informed consent were: Agreement to participate and decline to participate. Karolinska Ethics Committee approved the study protocol (approval nos. 2006/1273-31, 2014/2034-3). One patient declined to participate. Two patients were found to have microinvasive squamous cell carcinoma upon histopathological re-examination, when already enrolled in the study. These two patients were excluded from further follow-up analyses herein. Thus, the total number of patients in the present study is 529 .

1st follow-up visit: Gynecologic examination with colposcopy, clinician-collected cervical samples, other procedures. One of the two gynecologists (Dr. Andersson or Dr. Mints), who performed colposcopy and cervical sampling, first met with 
each patient. Punch biopsies were directed by colposcopy, and were obtained from visible lesions. The biopsies were histologically graded with the analysis done at Karolinska University Hospital. Standard procedures were followed, using the CIN classification (29). Patients in whom a recurrent lesion was found were sent for follow-up treatment. This was re-excision or simple total hysterectomy, based upon clinical evaluation and other considerations.

Enrolled women were followed according to national guidelines using cytology co-testing. The liquid-based method (ThinPrep ${ }^{\circledR}$, Hologic, Marlbororgh, MA, USA) was used for cytology and the Cobas 4800 assay (Roche Molecular Diagnostics, Pleasanton, CA, USA) for standard HPV testing. Samples were taken from the endocervix using cervical brushes and from the ectocervix with plastic spatulas. The samples were transferred into PreservCyt liquid-based cytology (LBC) vials according to European guidelines (30). The LBC was carried out at the Cytology Department of the Karolinska University Hospital, following the Bethesda system (31). The HPV DNA testing performed on-site was with the hospital's standard: Cobas 4800 HPV (Roche Diagnostics).

In addition, as part of the participation in the study, but not used for clinical decision-making, clinician-collected cervical samples (Abbott), self-collected vaginal samples (VSS) and urine samples were analyzed for comparative HPV testing at 1st follow-up. The procedure employed a multiplex real-time polymerase chain reaction (PCR) test which detects HPV16, HPV18, as well as other high-risk HPV: 31, 33, 35, 39, 45, 51, $52,56,58,59,66,68$. The results of this comparative testing are described in detail in a previous study (25). Herein, the results of the Abbott clinician-collected cervical samples and VSS are mainly presented for the patients in whom recurrent disease was detected.

Subsequent follow-up. Subsequent follow-up was based upon the results from LBC and Cobas HPV from the first follow-up. Insofar as cytological abnormalities were found, and/or the Cobas HPV result was positive, the patient was referred for a second follow-up. This second follow-up employed the same standard protocol as the first follow-up, and was based upon Swedish National Guidelines. The second follow-up was usually scheduled approximately one year after the first follow-up. Insofar as the cytology was negative for intraepithelial lesions or malignancy (NILM) and the HPV Cobas findings were also negative, Swedish National Guidelines were that the patient should return to routine triennial screening. This routine screening was envisioned to include HPV testing using Cobas from a clinician-taken sample and cytologic examination, with colposcopy performed according to clinician discretion.

Review of medical records. The entire medical record of each patient was thoroughly reviewed through December 2020. Information was obtained on age at the time of conization, the modality of conization, grade of dysplasia in the excised cone, number of cone pieces and margin status in the cone biopsy. Excisions were considered incomplete when dysplasia was found along the specimen margin, termed 'unclear' or when the margin status was uncertain. Assessment of the resection margins was further subdivided into: i) Ectocervical only, ii) endocervical only or iii) both margins unclear or uncertain. All comorbid diagnoses were noted. These were also categorized as conditions assumed to interact with HPV acquisition or CIN progression: autoimmune disorders, malignancy, infection with hepatitis or human immunodeficiency virus, diabetes mellitus, genetic disorders or organ transplantation $(19,26)$. Diagnosed recurrent/residual disease was defined as histologically-confirmed high-grade CIN on biopsy taken at colposcopy during any of the follow-up examinations.

Statistical analysis. A power analysis was performed prior to the study. Therein, it was estimated that 500 patients were needed for statistical significance at an alpha level of $\mathrm{P}<0.05$. Extensive univariate and bivariate analyses were first undertaken. The latter was performed using the Pearson $\chi^{2}$ test or Fisher's exact test if any expected cell was less than five, with one degree of freedom. All comparisons were two-sided. Salient dichotomizations were thereby made, as described in the Results section. Statistica (13.5.0.17/TIBCO-2018) and SPSS (IBM-version-25.0; IBM, Armonk, NY, USA) were used for statistical analysis. Multiple logistic regression was used to compute odds ratios (OR) and 95\% confidence intervals (CI) with the outcome being detected recurrence of high-grade CIN.

\section{Results}

Study overview and detection of recurrence. A summary of the number of women included in each step of the study is provided in Fig. 1. The detected recurrent cases are highlighted therein.

Demographic and other baseline univariate findings. Among the 529 patients included in the present study, the mean age was 34.3 years at the time of treatment. Altogether, 188 (35.5\%) of the patients were below age 30 at the time of treatment, and seventy-six patients (14.4\%) were age 45 or above at that time. Over two-thirds of the patients in this cohort were employed and well over half were university educated $(25,27,28)$.

Most of the patients (85\%) were treated using the contoured-loop excision of the transformation zone (C-LETZ) surgical method. Seventy-seven patients $(14.6 \%)$ were treated by laser conization, while three patients were treated with ablation. In the vast majority of the patients, a single cone piece was excised. The margin excision status revealed that the endocervical and ectocervical margins were both clear in $73 \%$ of the patients. Both these margins were either unclear or uncertain in $56(10.6 \%)$ patients, while in the remaining 87 patients only one of the margins was clear. The histology of the excised cone was CIN2 in 133 of the patients (25\%), CIN2/3 in 368 patients (70\%), CIN3/AIS in 15 patients and AIS in 13 patients.

Univariate findings from 1st follow-up. Over half the 529 patients came to 1st follow-up within six months after treatment; all but four of the patients came to 1st follow-up within one year after treatment. The latter four patients came within 15 months after treatment. At first follow-up the cytology results were available for all 529 patients; these were normal, NILM, in 453 (86\%) of the patients. There were also 


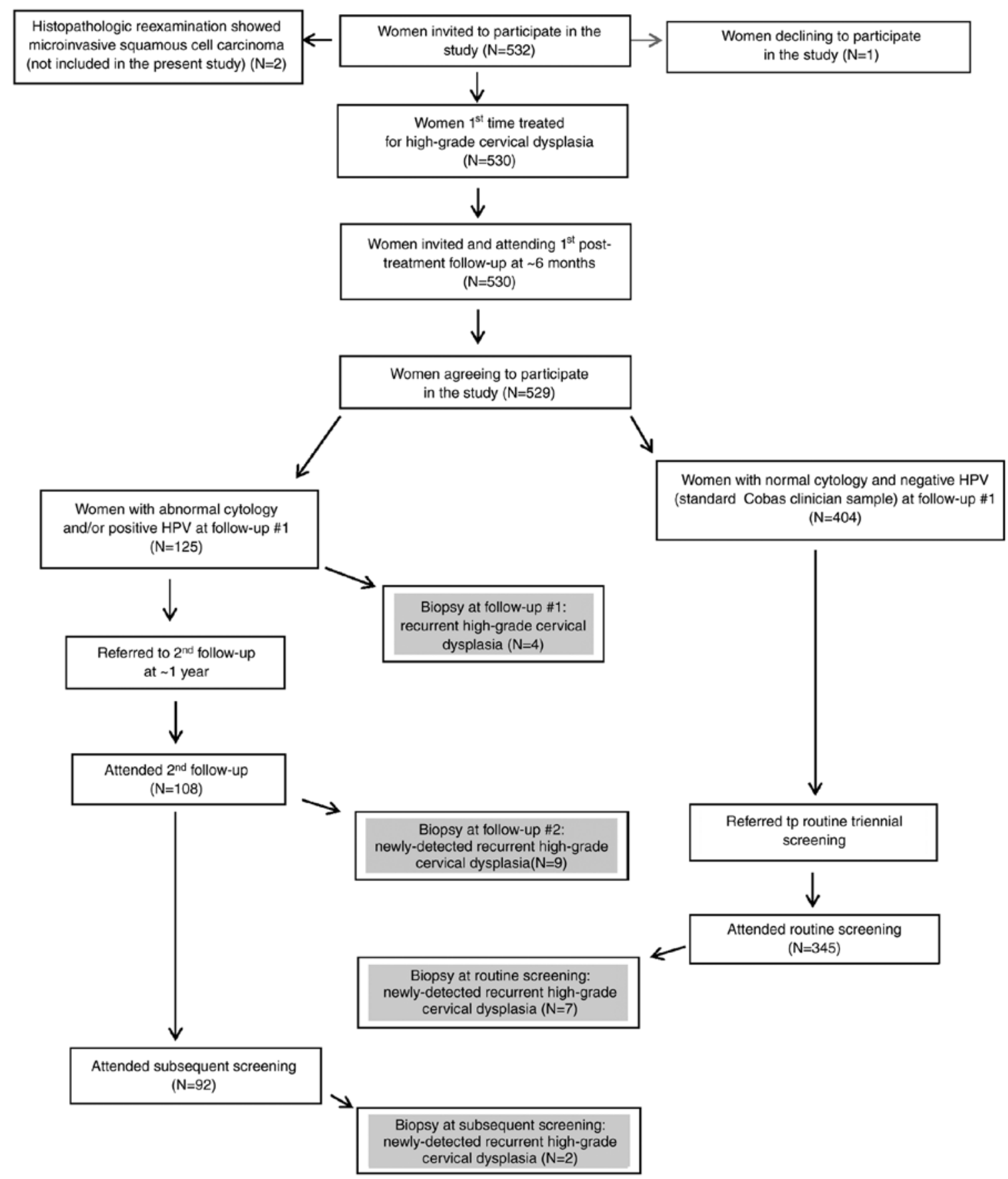

Figure 1. Flow chart of how the patients were triaged. The shaded rectangles highlight the patients in whom recurrence was detected in relation to the follow-up procedures. HPV, high-risk human papillomavirus.

complete data for HPV results at 1st follow-up; these were positive in $86(16.3 \%)$ of the patients. Altogether, 37 patients (7\%) had both abnormal cytology and HPV positive findings at 1 st follow-up. Four cases of recurrent high-grade CIN were detected at 1st follow-up.

Univariate findings from 2nd follow-up. Excluding the four patients in whom recurrence was detected at 1st follow-up, 121 patients of the 529 patients were referred to 2nd follow-up to which 108 of these patients attended. The majority of these patients attended 2nd follow-up within one year after the 1st follow-up; over $90 \%$ came within two years after the 1st follow-up. The longest time interval between the 1st and 2 nd follow-up was 3.5 years. At 2nd follow-up, 74 women had NILM, 31 had abnormal cytology and cytology was missing for three patients. Forty-three women had a positive HPV result and 51 had a negative HPV result at that time, while HPV data were not available for fourteen patients at 2 nd follow-up. Nine cases of recurrent high-grade CIN were detected at 2nd follow-up.

Univariate findings from routine follow-up. There were 404 women with normal cytology and negative HPV at 1st follow-up; these women were referred to routine triennial screening. Altogether 345 attended (85.4\%). Among the women who had attended 2nd follow-up without detected recurrence, 92 attended the subsequent routine screening. At 
the latter screening occasion, abnormal cytology was recorded in 34 women and NILM in 345, whereas cytology results were missing for 58 women who attended routine screening. The HPV results at routine follow-up were positive in 36 women, negative in 299 patients and were missing in 102 patients. Nine new cases of recurrent high-grade CIN were detected at routine follow-up.

Comorbidity. Altogether 136 patients (25.7\%) had one or more comorbid diagnosis reported in their medical records. Overall, the most frequent were psychiatric disorders in 51 patients, among whom 44 patients were noted to have clinical depression. Fifty-two patients $(9.8 \%)$ had a comorbid diagnosis assumed to interact with HPV acquisition or CIN progression, the most common being autoimmune conditions in 37 patients. The autoimmune conditions were varied and included rheumatoid arthritis, systemic lupus erythematosus, immune thrombocytopenia, hypothyroidism, inflammatory bowel disease and multiple sclerosis.

A comorbid malignancy was noted in ten patients (1.9\%). Two patients had had comorbid infectious disease associated with HPV acquisition/progression and two patients had type 2 diabetes mellitus. Genetic disorders were reported in four patients. Thirteen patients had two or more comorbid diagnoses reported in their medical records.

Detailed examination of the detected recurrent cases. Tables I, II and III provide an in-depth profile of each patient in whom recurrent high-grade CIN was detected at the 1st, 2nd or routine follow-up, respectively. Table I shows that all four recurrent cases detected at 1st follow-up were of squamous pathology on biopsy: high-grade squamous intraepithelial lesions (HSIL). A wide age distribution is observed among these patients. Only one of these four patients did not have clear ecto- and endocervical margins. All had HPV positive findings by the standard Cobas and Abbott clinician-taken samples, as well as from VSS. The patient with uncertain margins and one of the two patients in the age group between 56 and 60 also had HPV16 positive findings from clinician-taken samples and VSS, whereas none showed HPV18 positivity. The other patient within the 56 to 60 age group was the only one to have transformation zone 3 (TZ3) on colposcopy. She was also the only one in whom any comorbidity was reported, namely an autoimmune disorder and chronic obstructive pulmonary disease.

Table II is divided into two sub-parts: Table IIA presents the two patients in whom adenocarcinoma in situ, AIS, was found on biopsy at 2nd follow-up, while the seven patients with recurrent HSIL are presented in Table IIB. Both patients with recurrent AIS had TZ3 on colposcopy. The patient treated by laser conization had clear margins. By standard Cobas and VSS, the overall HPV results were negative at 1st follow-up. It was only by the clinician-sampling using the Abbott method that overall HPV positivity at 1st follow-up was revealed for this patient. The other patient with recurrent AIS had been treated by C-LETZ conization with an unclear endocervical margin. Whereas VSS had been negative, both clinician-sampling methods revealed HPV positivity. With the Abbott method, HPV18 was positive. At 2nd follow-up, HPV positivity was detected in both patients using the standard Cobas method.

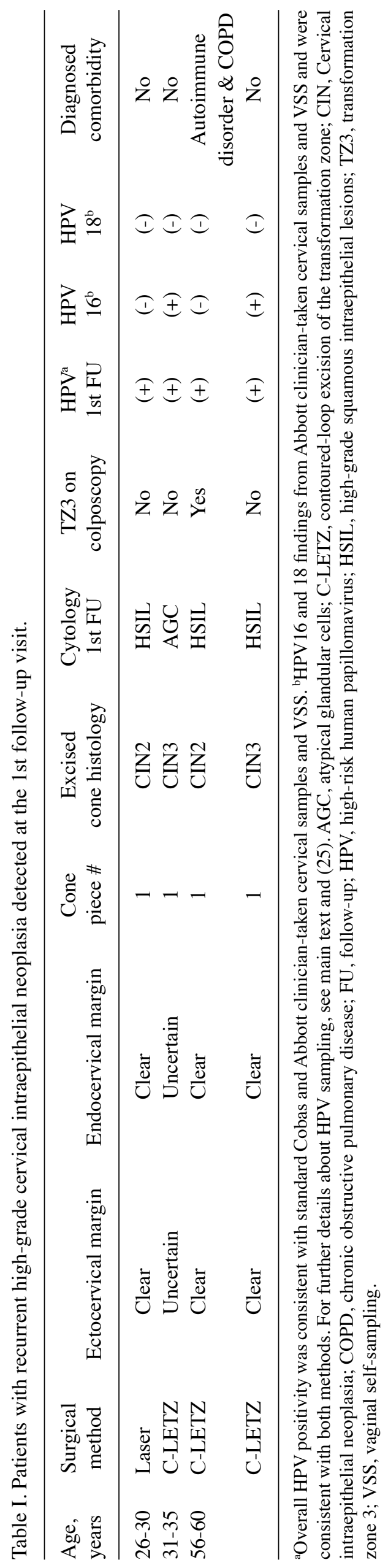









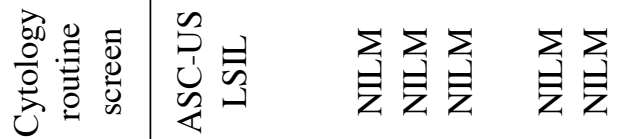

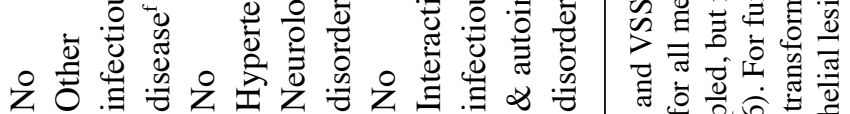

主言总总

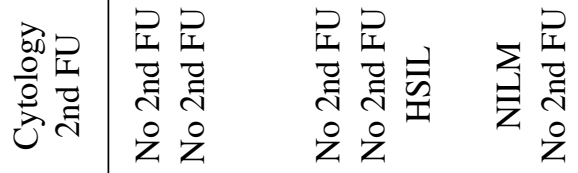

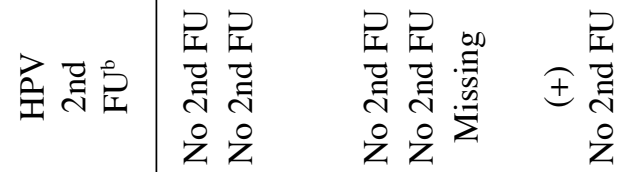

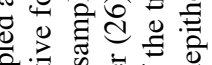

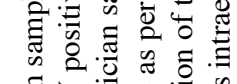

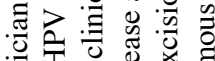

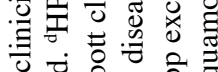

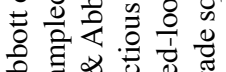

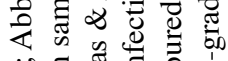

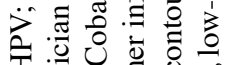

可言要的勻

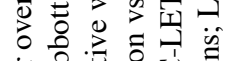

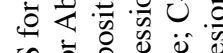

ᅰ

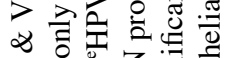

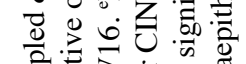

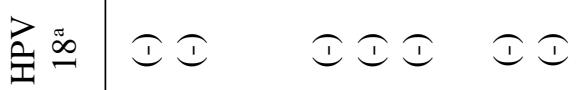

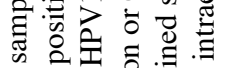

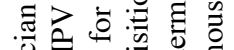

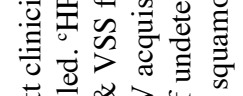

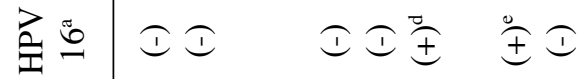

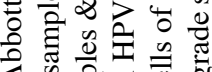

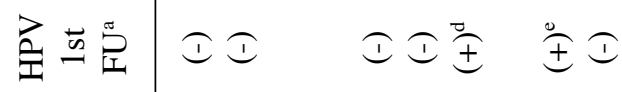

呵

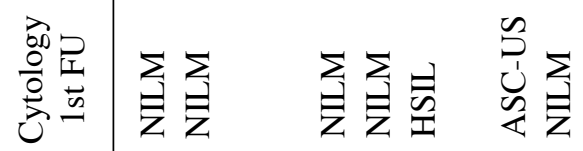

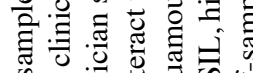

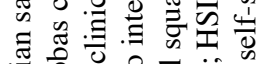

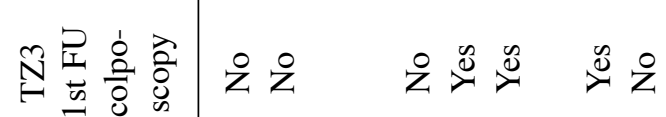

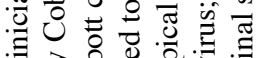

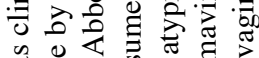

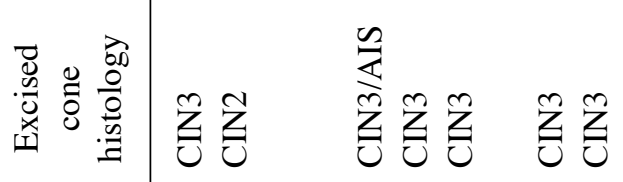

若

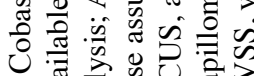

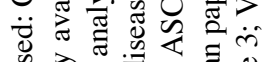

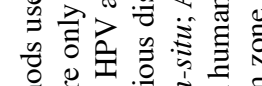

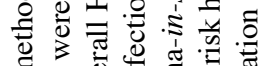

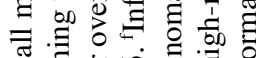

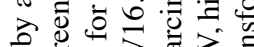

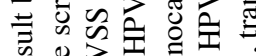

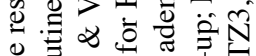

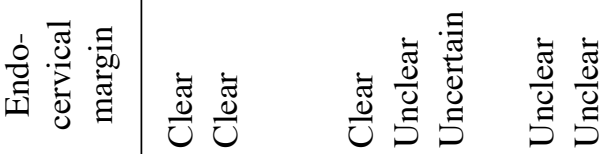

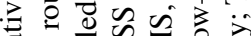

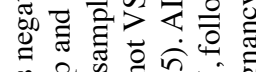

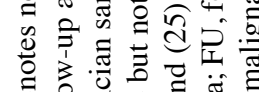

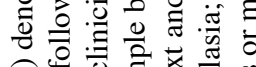

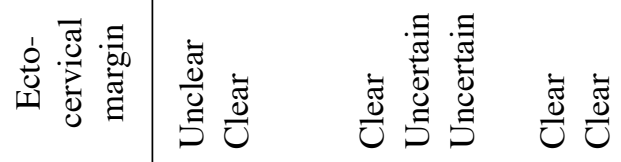

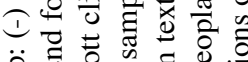

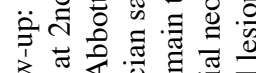

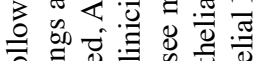

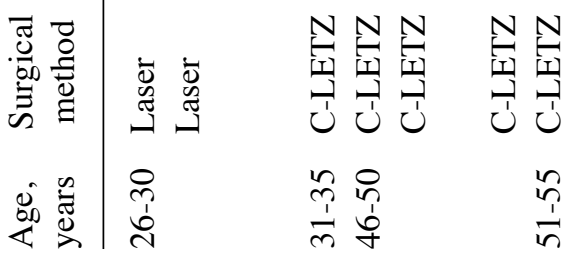

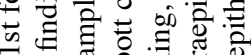

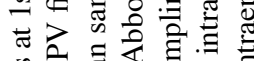

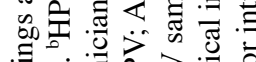

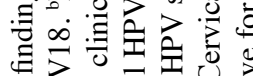

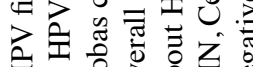


The seven patients with recurrent HSIL detected at 2nd follow-up also show a wide age distribution. All four patients above age 40 at the time of treatment had unclear or uncertain endocervical margins. One of them was treated by laser conization; another patient treated by C-LETZ had two cone pieces. All seven patients had HPV positive findings at 1st follow-up by all three methods. Positive HPV16 or HPV18 was found in four of these seven patients via VSS, while the clinician-taken sample did not reveal one case of HPV18 positivity. Among the six patients in whom this was assessed at 2nd follow-up, HPV was positive. Comorbidity (depression) was noted in only one of the nine patients with detected recurrence at 2 nd follow-up.

Table III is also divided into two sub-parts. In Table IIIA are the two patients with recurrent adenocarcinoma in situ, AIS, found at subsequent routine screening. All the margins had been uncertain in both cases. At 1st follow-up only Abbott clinician-samples were positive in one patient (HPV18); in the other patient all HPV testing was negative. Since cytology was NILM at that time, neither patient was referred to $2 \mathrm{nd}$ follow-up. It was only thereafter at routine screening that HPV on Cobas was positive and cytology revealed AIS. An autoimmune disorder was a diagnosed comorbidity in one of these patients.

Among the seven patients with recurrent HSIL (Table IIIB) detected at routine screening, five had NILM cytology and negative HPV via standard Cobas (as well as by Abbott clinician sample and VSS) at 1st follow-up and, therefore, were not referred to 2nd follow-up. In three of these patients, at least one margin had been unclear or uncertain. At subsequent routine screening, Cobas HPV was positive in three of these cases, but negative in one patient and missing in the other. The other two patients attended 2nd follow-up having had abnormal cytology at 1st follow-up as well as positive HPV at 1st follow-up. Neither of these patients had entirely clear margins at conization. Four of these seven patients had diagnosed comorbidity, including autoimmune and infectious diseases which are assumed to interact with HPV acquisition and/or CIN progression.

Bivariate analysis vis-à-vis detected recurrence of high-grade cervical dysplasia. In Table IV comparisons are made between the patients in whom recurrent high-grade cervical dysplasia was detected and those in whom recurrence was not detected. There were significantly more patients aged 45 or above with detected recurrence. Surgical method did not differ significantly between these two groups. However, $64 \%$ of the patients with detected recurrence had at least one unclear or uncertain margin, whereas this was the case for $25 \%$ of the patients without detected recurrence. Neither the number of cone pieces nor the histology of the excised cone differed significantly for the patients with vs. without diagnosed recurrence. Comorbidity did not significantly distinguish these two groups, nor did the finding of TZ3 on colposcopy at 1st follow-up.

Cytology at 1st follow-up was significantly more often abnormal among the patients with detected recurrence at any of the follow-up times (59\%) compared to those in whom no recurrence was detected (12\%). Excluding the four patients in whom recurrence was detected at 1st follow-up, abnormal cytology at 2nd follow-up was significantly more frequent among patients with detected recurrence (91\%) vs. those in whom no recurrence was detected (22\%). Among the nine patients in whom recurrence was detected at subsequent follow-up, four (44.4\%) had abnormal cytology at that follow-up occasion, whereas $30(8.1 \%)$ of the 370 patients without detected recurrence in whom cytology was reported at subsequent follow-up had an abnormal result.

Likewise, standard Cobas HPV was significantly more often positive at each follow-up among patients with detected recurrence compared to those in whom no recurrence was detected. At 1st follow-up, $64 \%$ of the 22 patients in whom recurrence was detected showed HPV positivity, vs. $14 \%$ among the 507 patients without detected recurrence. Excluding the four patients in whom recurrence was detected at 1st follow-up, at 2nd follow-up, all nine patients with detected recurrence in whom these results were available showed HPV positivity. In contrast, 34 of the 85 patients without detected recurrence in whom HPV results were available at 2nd follow-up showed HPV positivity at that occasion. HPV results were available for seven of the nine patients in whom recurrence was detected at subsequent routine follow-up, and were positive in six cases $(85.7 \%)$. In contrast, among the 328 patients without detected occurrence in whom these HPV was assessed at routine follow-up, only 30 patients showed HPV positivity.

The limited available data on persistent HPV positivity indicates that all eight patients with detected recurrence and HPV positivity with standard Cobas at 1st follow-up also showed positivity at 2 nd follow-up. In contrast, nearly $50 \%$ of the patients without detected recurrence who had HPV positive results with standard Cobas at 1st follow-up had negative HPV at 2nd follow-up. Of the nine patients in whom recurrence was detected at subsequent follow-up, one had HPV positive findings with standard Cobas at 1st follow-up and at subsequent follow-up. Among those without detected recurrence, there were 15 patients with HPV positivity both at 1st follow-up and at routine screening, while 36 patients had HPV positive findings at 1st follow-up but not at routine screening.

Age-related bivariate findings. The age-related findings are presented in Table $\mathrm{V}$, with stratification of the patients below age 30 , those aged 30 to 44 and patients aged 45 or above. The statistical analysis compares all patients below age 45 with those aged 45 or above. Firstly, it is seen that significantly more of the patients aged 45 or above were treated by the laser method (24\%) compared to $13 \%$ among those below age 45 . There were no significant age-related differences regarding margin excision status.

A highly significant difference was noted regarding the prevalence of TZ3 on colposcopy $(67 \%)$ at 1st follow-up among patients aged 45 or above vs. $36 \%$ for patients below age 45 . Patients aged 45 or above significantly more often had abnormal cytology at 1st follow-up (24\%) vs. $13 \%$ seen in patients younger than 45 . Further analysis among the eighteen patients age $\geq 45$ with abnormal cytology at 1 st follow-up reveals that six patients had HSIL, four had low-grade squamous intraepithelial lesions (LSIL) and eight had atypical squamous cells of undetermined-significance (ASC-US). In contrast, ten patients below age 45 had HSIL, one patient had ASC-H (atypical squamous cells cannot exclude HSIL) and 
Table IV. Comparison of the patients with and without detected recurrence of high-grade cervical intraepithelial neoplasia.

\begin{tabular}{|c|c|c|c|}
\hline Variable & $\begin{array}{c}\text { No recurrence of } \\
\text { high-grade CIN detected }\end{array}$ & $\begin{array}{c}\text { Recurrent } \\
\text { high-grade CIN detected }\end{array}$ & P-value \\
\hline Age at conization, years & & & $<0.01$ \\
\hline$<45$ & 440 & 13 & \\
\hline$\geq 45$ & 67 & 9 & \\
\hline Surgical method & & & NS \\
\hline C-LETZ & 432 & 17 & \\
\hline Laser & 72 & 5 & \\
\hline Ablation $^{\mathrm{a}}$ & 3 & & \\
\hline Margin excision status $^{b}$ & & & $<0.001$ \\
\hline Both margins clear & 378 & 8 & \\
\hline Only ectocervical margin unclear/uncertain & 41 & 1 & \\
\hline Only endocervical margin unclear/uncertain & 38 & 7 & \\
\hline Both margins unclear/uncertain & 50 & 6 & \\
\hline Number of cone pieces ${ }^{\mathrm{a}}$ & & & NS \\
\hline One & 416 & 18 & \\
\hline Two or more & 88 & 4 & \\
\hline Histology of excised cone & & & NS \\
\hline CIN2 & 128 & 5 & \\
\hline CIN2/3 or worse & 379 & 17 & \\
\hline Any diagnosed comorbidity & & & NS \\
\hline No & 378 & 15 & \\
\hline Yes & 129 & 7 & \\
\hline Any diagnosed comorbidity linked to HPV or CIN progression ${ }^{c}$ & & & NS \\
\hline No & 458 & 19 & \\
\hline Yes & 49 & 3 & \\
\hline Diagnosed comorbid malignancy & & & NS \\
\hline No & 497 & 22 & \\
\hline Yes & 10 & 0 & \\
\hline Colposcopy at 1 st follow-up: $\mathrm{TZ}^{\mathrm{d}}$ & & & NS \\
\hline No & 298 & 14 & \\
\hline Yes & 205 & 8 & \\
\hline Cytology at 1st follow-up & & & $<0.001$ \\
\hline NILM & 444 & 9 & \\
\hline Abnormal & 63 & 13 & \\
\hline Cytology at 2nd (referred) follow-up ${ }^{\mathrm{e}, \mathrm{f}}$ & & & $<0.001$ \\
\hline NILM & 73 & 1 & \\
\hline Abnormal & 21 & 10 & \\
\hline Cytology at routine follow-up ${ }^{\mathrm{e}, \mathrm{g}}$ & & & $<0.01$ \\
\hline NILM & 340 & 5 & \\
\hline Abnormal & 30 & 4 & \\
\hline HPV at 1 st follow-up (Standard Cobas) & & & $<0.001$ \\
\hline Negative & 435 & 8 & \\
\hline Positive & 72 & 14 & \\
\hline HPV at 2nd (referred) follow-up (Standard Cobas) $)^{\mathrm{e}, \mathrm{h}}$ & & & $<0.001$ \\
\hline Negative & 51 & 0 & \\
\hline Positive & 34 & 9 & \\
\hline HPV at routine follow-up (Standard Cobas) ${ }^{\mathrm{e}, \mathrm{i}}$ & & & $<0.001$ \\
\hline Negative & 298 & 1 & \\
\hline Positive & 30 & 6 & \\
\hline
\end{tabular}


Table IV. Continued.

\begin{tabular}{|c|c|c|c|}
\hline Variable & $\begin{array}{l}\text { No recurrence of } \\
\text { high-grade CIN detected }\end{array}$ & $\begin{array}{l}\text { Recurrent } \\
\text { high-grade CIN detected }\end{array}$ & P-value \\
\hline Persistent HPV positivity (Standard Cobas) $)^{\mathrm{e}, \mathrm{j}}$ & & & $<0.01$ \\
\hline $\begin{array}{l}\text { No (HPV positive at 1st follow-up, HPV negative at } \\
\text { 2nd follow-up) }\end{array}$ & 27 & 0 & \\
\hline Yes (HPV positive at 1st \& 2nd follow-up) & 28 & 8 & \\
\hline Persistent HPV positivity (Standard Cobas) $)^{\mathrm{e}, \mathrm{k}}$ & & & NS \\
\hline $\begin{array}{l}\text { No (HPV positive at 1st follow-up, HPV negative at } \\
\text { routine follow-up) }\end{array}$ & 36 & 0 & \\
\hline Yes (HPV positive at 1st follow-up \& routine follow-up) & 15 & 1 & \\
\hline \multicolumn{4}{|c|}{ 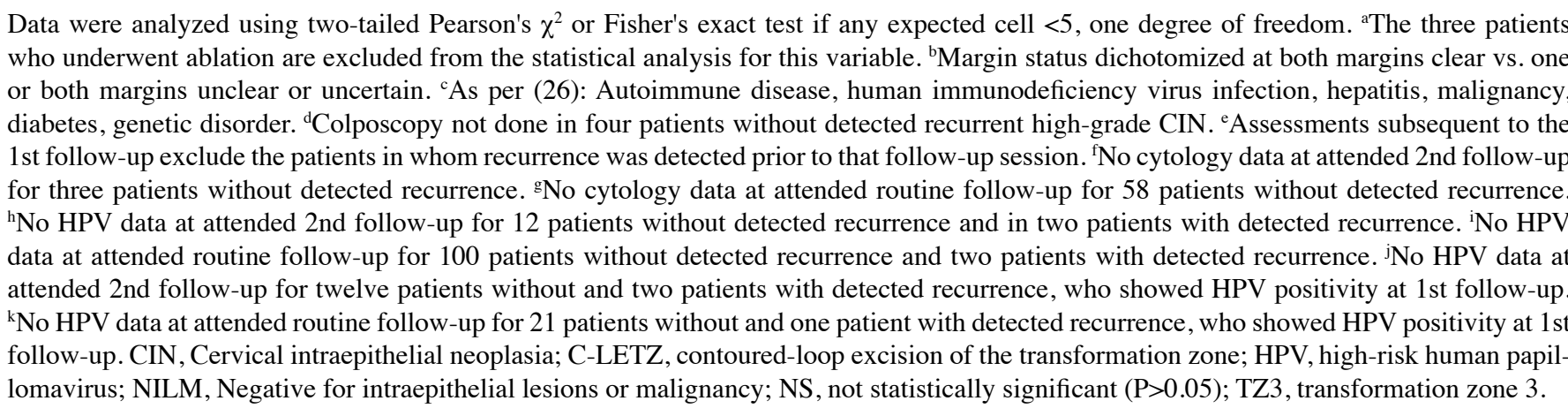 } \\
\hline
\end{tabular}

23 had LSIL. Atypical glandular cells (AGC) were found in five patients below age 45. Twenty-five percent of the patients aged 45 or above were found to have HPV positivity using the standard Cobas method at 1st follow-up. This was significantly more than among those below age $45(15 \%)$.

Approximately $12 \%$ of the patients had no further follow-up after the 1st follow-up, with a fairly similar percentage in each of the age groups. At 2 nd and routine follow-up, there were no significant age-related differences in cytology or HPV.

Borderline significantly more patients aged 45 or above had any diagnosed comorbidity. Five of the 76 patients aged 45 or above had a diagnosed comorbid cancer $(6.6 \%)$ vs. $1.1 \%$ in the younger patients.

The previously noted significant age-related difference in detected recurrence rate is presented in more detail here in Table V. Recurrence was detected in $2.1 \%$ of the patients below age $30,3.4 \%$ in those aged 30 to 44 and $11.8 \%$ for patients aged 45 or above.

Bivariate findings vis-à-vis margin status. The focus of Table VI is on margin status. For statistical analysis, this is dichotomized as both margins clear vs. any or both margins unclear or uncertain. The margin status did not differ significantly in relation to the surgical method employed. However, with more than one cone piece there was a significantly greater likelihood that at least one margin was unclear or uncertain compared to when only one cone piece was taken. Further analysis reveals that although the surgical method was not significantly associated with margin status, there was a greater likelihood of more than one cone piece with C-LETZ compared to laser surgical method (Pearson $\chi^{2}=13.9$, $\mathrm{P}=0.0002)$. There were only two cases in which more than one cone piece was reported with laser, whereas in 90 cases using C-LETZ more than one cone piece was reported. In $85 \%$ of cases with one or more margins uncertain or unclear, the histology of the excised cone was CIN2/3 or worse. More severe cone histology was significantly less frequent $(71 \%)$ when both margins were clear.

Abnormal cytology at 1st follow-up was significantly more common when one or more margin was unclear or uncertain. Although there was a greater percentage of HPV positivity with one or both unclear margins, HPV positivity at 1st follow-up did not significantly differ according to margin status. As noted, at least one of these abnormal findings was needed to refer the patient to 2 nd follow-up. At the bottom of Table VI, it is seen that there was no significant association between abnormal cytology and/or positive HPV at 1st follow-up and unclear or uncertain margins. Altogether 103 patients with at least one unclear or uncertain margin had negative HPV and normal cytology at 1st follow-up. According to the current protocol, these 103 patients were returned to routine screening.

Multiple logistic regression findings with detected recurrence as the outcome. Table VII displays three multiple logistic regression models with detected recurrence of high-grade CIN as the outcome. All the models are highly statistically significant and the data are complete, i.e. all 529 patients are included in the analysis. The first model includes four independent variables that each significantly predicts detected recurrence. These predictors are age 45 or above at the time of conization, incomplete lesion excision, i.e. one or both unclear or uncertain margins at conization, positive HPV at 1st follow-up using the standard Cobas clinician-taken sample and abnormal cytology 


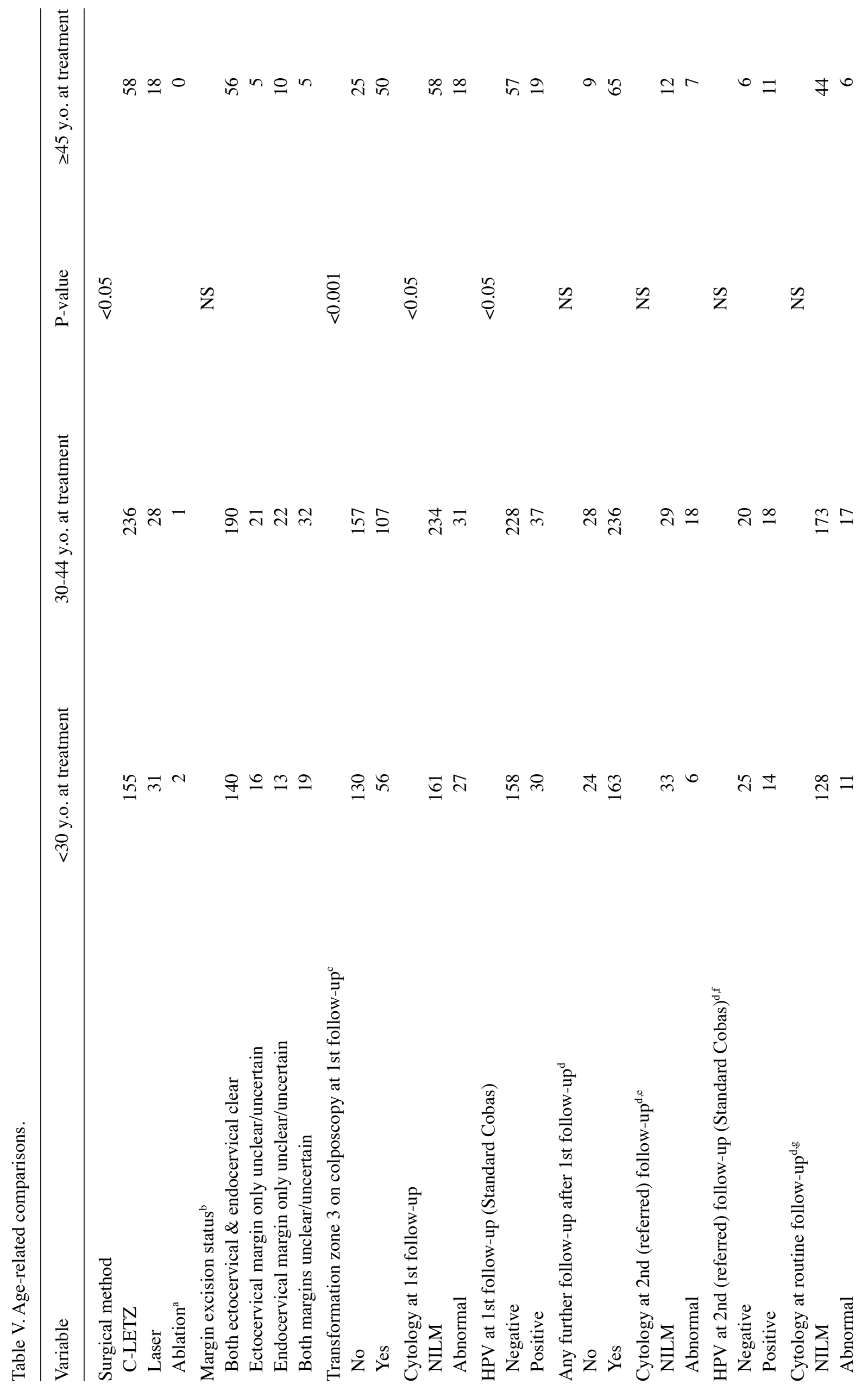




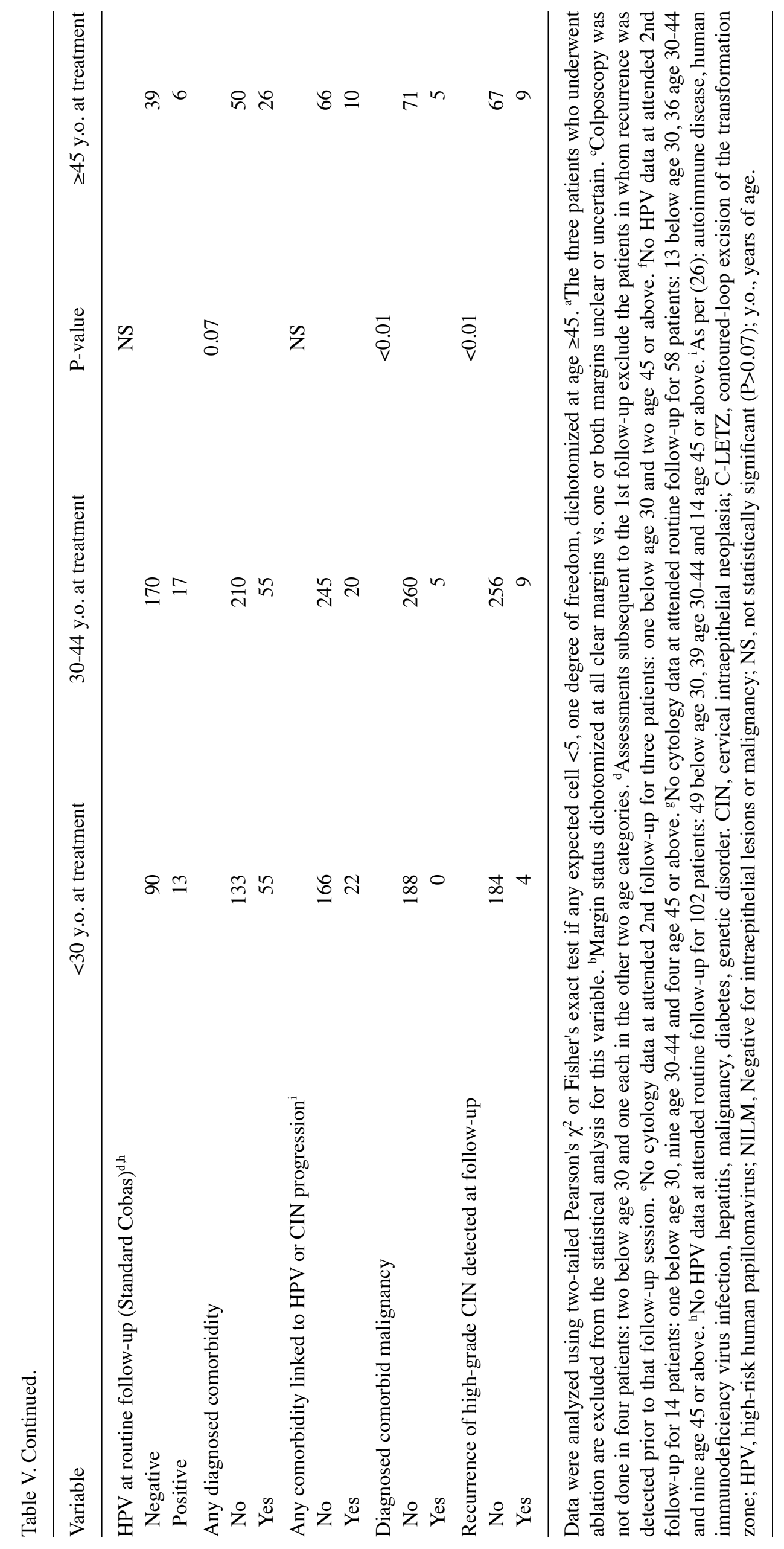


Table VI. Comparisons related to margin status.

\begin{tabular}{|c|c|c|c|c|c|}
\hline Variable & $\begin{array}{l}\text { Both } \\
\text { margins } \\
\text { clear }\end{array}$ & P-value & $\begin{array}{l}\text { Only } \\
\text { ectocervical margin } \\
\text { unclear/uncertain }\end{array}$ & $\begin{array}{c}\text { Only } \\
\text { endocervical margin } \\
\text { unclear/uncertain }\end{array}$ & $\begin{array}{c}\text { Both margins } \\
\text { unclear/uncertain }\end{array}$ \\
\hline Surgical method & & NS & & & \\
\hline C-LETZ & 329 & & 33 & 36 & 51 \\
\hline Laser & 54 & & 9 & 9 & 5 \\
\hline Ablation $^{\mathrm{a}}$ & 3 & & 0 & 0 & 0 \\
\hline Cone pieces ${ }^{\mathrm{a}}$ & & $<0.01$ & & & \\
\hline Only one & 326 & & 34 & 40 & 34 \\
\hline More than one & 57 & & 8 & 5 & 22 \\
\hline Histology of excised cone & & $<0.01$ & & & \\
\hline CIN2 & 112 & & 8 & 5 & 8 \\
\hline CIN2/3 or worse & 274 & & 34 & 40 & 48 \\
\hline Cytology at 1 st follow-up & & $<0.05$ & & & \\
\hline NILM & 338 & & 39 & 32 & 44 \\
\hline Abnormal & 48 & & 3 & 13 & 12 \\
\hline HPV at 1 st follow-up (Standard Cobas) & & NS & & & \\
\hline Negative & 326 & & 40 & 34 & 43 \\
\hline Positive & 60 & & 2 & 11 & 13 \\
\hline $\begin{array}{l}\text { Cytology \& HPV (Standard Cobas) at } \\
\text { 1st follow-up }\end{array}$ & & NS & & & \\
\hline NILM and negative HPV & 301 & & 37 & 29 & 37 \\
\hline Abnormal cytology \&/or positive HPV & 85 & & 5 & 16 & 19 \\
\hline
\end{tabular}

Pearson's $\chi^{2}$ 2-tailed P-values with one degree of freedom with margin status dichotomized at both margins clear vs. one or both margins unclear or uncertain. ${ }^{a}$ The three patients who underwent ablation are excluded from the statistical analysis for this variable. CIN, cervical intraepithelial neoplasia; C-LETZ, contoured-loop excision of the transformation zone; HPV, high-risk human papillomavirus; NILM, Negative for intraepithelial lesions or malignancy.

at 1st follow-up. The second model includes one more independent variable, any diagnosed comorbidity, which did not significantly predict detected recurrence. This second model had only a slightly higher overall $\chi^{2}$ than the first model with one less independent variable. In the third model, comorbidity with conditions that have been linked to HPV acquisition and/or CIN progression was included, rather than any diagnosed comorbidity. The latter independent variable yielded a slightly higher odds ratio, but still non-significant prediction of detected recurrence.

Adjusting for all the other significant independent variables, i.e. age, HPV status at 1st follow-up and abnormal cytology, we performed further multiple logistic regression. It was found that only unclear or uncertain ectocervical margins were non-significant for predicting detected recurrence. That analysis included 428 patients and there was only one patient with detected recurrence who had only unclear or uncertain ectocervical margins. In contrast, however, only unclear or uncertain endocervical margins did significantly predict detected recurrence $[\mathrm{OR}=6.2$ (95\% CI: 1.8-21.4) $\mathrm{P}=0.004]$. Altogether 431 patients were included in that analysis, with seven detected recurrences among those with only unclear or uncertain endocervical margins. When both margins were unclear or uncertain, the
OR was 5.5 (95\% CI: 1.6-18.9, $\mathrm{P}=0.006)$ for detected recurrence. There were six detected recurrences among those with both margins unclear or uncertain, and the overall analysis included 442 patients.

\section{Discussion}

A unique feature of the present study is practically complete attendance to early follow-up after treatment of high-grade CIN for a sizable cohort of patients, coupled with complete data vis-à-vis HPV status and cytology plus nearly complete colposcopy data at that early follow-up occasion. In addition, complete information was obtained about diagnosed comorbidity. These unique features are combined with full data on surgical method, margin excision status and histology of the excised cone. After first follow-up, the patients were triaged as per national guidelines, with subsequent data available on a very large percentage, $88.4 \%$, of this cohort who continued to attend follow-up as per recommendation. In other words, this study combines the ideal situation of complete early follow-up with a clearly-described situation thereafter, for at least four years, up to a maximum of more than six years. During that period, recurrent high-grade CIN was detected in altogether twenty-two patients. 
Table VII. Prediction of detected recurrent high-grade cervical intraepithelial neoplasia in 529 treated patients assessed via multiple logistic regression.

\begin{tabular}{|c|c|c|c|c|c|}
\hline Model $\chi^{2}$ & Variable & OR & $-95 \% \mathrm{CI}$ & $+95 \% \mathrm{CI}$ & P-value \\
\hline \multirow[t]{4}{*}{$53.2^{\mathrm{a}}$} & Age 45 or above & 3.5 & 1.3 & 9.9 & $<0.05$ \\
\hline & One or more unclear or uncertain margins & 5.3 & 2 & 14.2 & $<0.001$ \\
\hline & HPV positive at 1 st follow-up (Standard Cobas) & 5.8 & 2 & 16.8 & $<0.01$ \\
\hline & Abnormal cytology at 1 st follow-up & 3.9 & 1.4 & 11 & $<0.05$ \\
\hline \multirow[t]{5}{*}{$53.3^{\mathrm{a}}$} & Age 45 or above & 3.4 & 1.2 & 9.6 & $<0.05$ \\
\hline & One or more unclear or uncertain margins & 5.4 & 2 & 14.5 & $<0.001$ \\
\hline & HPV positive at 1 st follow-up (Standard Cobas) & 5.8 & 2 & 16.7 & $<0.01$ \\
\hline & Abnormal cytology at 1 st follow-up & 3.9 & 1.4 & 11.2 & $<0.05$ \\
\hline & Any diagnosed comorbidity & 1.3 & 0.4 & 3.6 & NS \\
\hline \multirow[t]{5}{*}{$53.4^{\mathrm{a}}$} & Age 45 or above & 3.4 & 1.2 & 9.6 & $<0.05$ \\
\hline & One or more unclear or uncertain margins & 5.4 & 2 & 14.6 & $<0.001$ \\
\hline & HPV positive at 1st follow-up (Standard Cobas) & 5.8 & 2 & 16.8 & $<0.01$ \\
\hline & Abnormal cytology at 1 st follow-up & 3.8 & 1.3 & 11 & $<0.05$ \\
\hline & Any diagnosed comorbidity linked to HPV or to CIN progression ${ }^{\mathrm{b}}$ & 1.5 & 0.4 & 5.8 & NS \\
\hline
\end{tabular}

The data are complete for all the predictor variables and the outcome: Detected recurrent high-grade CIN ( $\mathrm{n}=22)$ vs. no detected recurrence $(\mathrm{n}=507)$. ${ }^{\mathrm{a}}$ Model $\chi^{2} \mathrm{P}<0.0001 .{ }^{\mathrm{b}} \mathrm{As}$ per (26): Autoimmune disease, human immunodeficiency virus infection, hepatitis, malignancy, diabetes, genetic disorder. CI, confidence interval; CIN, cervical intraepithelial neoplasia; HPV, high-risk human papillomavirus; NS, statistically non-significant $(\mathrm{P} \geq 0.05)$; OR, odds ratio.

Given the completeness of the early follow-up data, powerful multivariate models could be generated to identify four significant independent risk factors for detected recurrence of high-grade CIN. These were: age 45 or above at the time of conization, one or more unclear or uncertain excision margins, positive HPV and abnormal cytology at 1st follow-up. In contradistinction to previous long-term findings (19), diagnosed comorbidity which is potentially linked to HPV or to CIN progression was not found to significantly predict detected recurrence in the present study. Surgical method and histology of the excised cone, likewise, were not found to be directly associated with increased detected recurrence risk, similarly to other investigations $(19,32)$.

The strong association between margin status and recurrence risk found herein is consistent with a number of other studies (19-23). Moreover, the findings that incomplete excision from the endocervical or both margins, but not from ectocervical margins alone show significant multivariate association with detected recurrence, are consistent with other investigations $(19,20,33)$. Of particular note is that in the present study, this increased risk is not significantly associated with HPV positivity at early follow-up. Moreover, patients with negative HPV and NILM cytology at 1st follow-up were thereafter referred to routine triennial screening, at which time five of the seven patients in whom recurrence was detected had at least one unclear or uncertain margin. Incomplete resection was significantly more likely with more severe cone histology. The latter, however, in itself, did not show a direct relation with recurrence.

Although, overall, there was no significant association found between age and margin status, most of the patients aged 45 or above in whom recurrence was detected also had at least one unclear or uncertain margin. Specifically, the margins were reportedly clear only in the two patients above age 45 with recurrence detected at 1st follow-up. The seven other patients aged 45 or above in whom recurrence was subsequently detected all had at least one unclear or uncertain margin. Thus, the present findings would seem to support the recommendation $(20,21)$ that larger and thereby more complete excisions should be made when treating women in the post-menopausal age group with high-grade CIN.

Significantly more women aged 45 or above were treated with laser rather than with contoured loop excision of the transformation zone, C-LETZ. Although surgical method did not show a significant association with detected recurrence, with the laser technique, there was a significantly greater likelihood that only one cone piece would be generated. In turn, with a single cone piece, the chances of having clear margins was significantly greater. It should also be noted that none of the women aged 45 or above with detected recurrence had been treated with laser. Although the laser technique is a higher-cost procedure requiring particular colposcopic expertise $(34,35)$, it might be inferred from the results of the present study that the laser technique would be a good option for women in this age group.

Concordant with other reports (36), in the present study, the patients near or in the post-menopausal age group, i.e. 45 or above, significantly more often had the finding of transition zone $3, \mathrm{TZ} 3$, on colposcopy compared to the women below age 45. This finding indicates that the squamo-columnar junction is located in the endocervix, thereby resulting in an unsatisfactory colposcopic examination (37). Although colposcopy was performed at 1st follow-up in all but four of the 529 patients, biopsies were only taken from visible lesions, which were very few, thirteen altogether. High-grade cervical dysplasia may 
also be found on biopsies taken from colposcopically negative sites (38). It can therefore be surmised that the actual number of recurrences may have been underestimated (25). This underestimation could well have been even more pronounced among patients aged 45 or above due to the higher percentage of TZ3 on colposcopy.

Patients aged 45 or above significantly more often had abnormal cytology and positive HPV at 1st follow-up, compared to those younger than 45 . Nevertheless, fifty-one patients $(67 \%)$ of the seventy-six patients aged 45 or above had NILM on cytology and negative HPV at 1st follow-up, and were therefore referred directly to routine triennial screening, according to national guidelines. At that screening occasion, recurrence was detected in two more patients above age 45 , both with unclear endocervical margins but with NILM and negative HPV at 1st follow-up. Notably, one of these two patients also had two comorbid diagnoses (infectious disease and autoimmune disorder) that have been linked to HPV and CIN progression $(19,26)$. This patient also had a positive HPV finding at the subsequent routine screening.

With complete data on HPV as well as cytology at 1 st follow-up for all 529 patients, a fuller picture emerges of the major contributors to recurrent high-grade CIN. Abnormal cytology slightly surpassed age in its power to predict recurrence. A positive HPV result is seen as a very powerful predictor, with a higher odds ratio, although lower statistical significance than margin status.

Positive HPV as well as abnormal cytology at 2nd follow-up and at routine follow-up each also showed significant association with detected recurrence in bivariate analysis. The importance of persistent HPV positivity after treatment of high-grade CIN has been emphasized (39). Patterns of persistent HPV infection after treatment of high-grade CIN were the focus of a previous study (9) since persistence is considered to be the key contributor of progression to invasive cervical cancer. The difficulties in finding the optimal protocol for assessing post-therapeutic HPV persistence were underscored in a previous study (9). In the present study, the patients with positive HPV at 1st follow-up were referred to 2nd follow-up. As seen from Table IV, 57\% of the patients at 2nd follow-up had persistent HPV findings, with significantly more persistent HPV among the patients in whom recurrence was detected. However, there was some loss to follow-up among the 125 patients who were referred to 2 nd follow-up. Moreover, at 2nd follow-up and particularly at subsequent routine screening, there were substantial missing HPV data. As shown in Table IIIB, regarding the two patients with detected recurrence at routine screening who had also attended 2nd follow-up, complete HPV data indicating persistent HPV positivity at all three occasions was seen in only one patient. These subsequent HPV data were missing in the other patient.

Heretofore in the present paper, the main focus vis-à-vis the HPV results has been on the clinician-taken cervical samples via the standard Cobas method. Albeit missing in a few of the patients who attended 2 nd follow-up and in a substantial number of those who attended routine follow-up, these results could be assessed and compared across the three screening occasions. As described in detail in a previous study (25), at 1st follow-up complete data were also available for vaginal self-taken samples, VSS, and clinician-taken cervical samples via the Abbott method. Based on the results available at that time for 1st and 2nd follow-up, overall HPV positivity was found by each of these three methods in all eleven patients in whom recurrence was detected and who had squamous pathology. However, in the remaining two patients in whom the recurrent pathology was glandular, HPV positivity was not detected by VSS, but only by clinician-taken cervical samples (These findings are shown in Tables I and II). In both patients with glandular pathology, HPV18, an especially potent risk indicator $(40,41)$, was found to be positive with clinician sampling. Those limited data seemed to suggest that self-sampling should not be recommended for patients with glandular pathology, but only for those in whom the pathology was squamous (25).

We can herein ask whether more insights are gleaned from the additional follow-up data for subsequent routine screening. With respect to the two patients in whom recurrent glandular pathology was detected at that later time (Table IIIA), concordantly, vaginal self-sampling did not reveal a positive HPV result. In one patient only the Abbott clinician-taken sample (not Cobas) was positive and, moreover, indicated HPV18 positivity. In the other patient with glandular pathology, however, HPV positivity was not detected in either of the clinician-sampled cervical specimens at 1st follow-up. We herein describe the additional analysis of the 27 patients without detected recurrence in whom there was glandular histology in the excised cone and/or AGC on cytology at any of the follow-up periods. This reveals that three patients showed positivity on both Abbott clinician-taken samples and VSS, in 22 patients both were HPV negative, one patient showed HPV positivity only on VSS and in one patient only the clinician sample was positive. Comparing VSS and Cobas clinician-taken samples shows nearly identical findings, except that there were 23 patients with HPV negative on both, and in no case was Cobas positive when VSS was negative. Taken together, these results seem to suggest that VSS may not be inferior to clinician-sampling for follow-up of patients with glandular pathology. Further study of this question is needed.

Scrutiny is warranted, as well, of the patients with squamous pathology in whom recurrence was detected at subsequent routine follow-up. The two patients who also attended 2 nd follow-up had positive HPV in both clinician-taken samples, while VSS was positive in only one of these cases. Among the five patients with recurrent squamous pathology and who only attended subsequent routine screening, HPV at 1st follow-up was negative not only by the standard Cobas, but also from the Abbott clinician-taken samples and VSS. The overriding conclusion from analyzing all nine recurrent cases detected at subsequent routine follow-up is that repeated HPV testing is indispensable for adequate follow-up of patients treated for high-grade cervical intraepithelial dysplasia. Assessment of high-risk subtypes HPV16 and 18 is particularly advisable.

Besides the missing HPV data for 102 of the 437 patients who came to routine follow-up (23\%), 61 patients did not attend any subsequent follow-up after the 1st one. This is of major concern, given the elevated risk of recurrent high-grade cervical intraepithelial dysplasia and invasive cervical cancer. Home self-sampling for HPV could well be a realistic solution. Our earlier study among 479 women from the present cohort treated for high-grade CIN indicated that the vast 
majority were amenable to performing self-sampling at home and considered self-sampling to have been implemented without difficulty (27). Notably, self-sampling in our study was performed in the clinic restroom, which was less comfortable than the home environment (25).

In the most recent period through the end of the year 2020 for which we have follow-up data on the present cohort, a new situation arose due to the global COVID-19 pandemic. In Sweden, during the 1st wave of the COVID epidemic, cervical screening was largely cancelled, including the cancellation of 192000 cervical screening appointments in Stockholm (42). Concordantly, a number of reports worldwide indicate a precipitous decline in cervical screening (43-47). The actual and potential consequences of the COVID-19 pandemic vis-à-vis increased cervical cancer incidence, treatment delay and mortality have been underscored $(45,48,49)$. Home self-sampling for HPV is widely endorsed as the key strategy to avoid these pandemic-related, adverse consequences (42,44,47,50-53). As succinctly stated in a previous study (50): 'The new imperatives of the COVID-19 pandemic support self-sampled HPV testing as the primary cervical screening method'. That conclusion is justified, given the reliability of self-sampling (25,54-57), together with its acceptability and cost-effectiveness (27,58-63).

Considering the elevated risk of recurrence associated with increased age and the importance of persistent HPV infection, home self-sampling for HPV could be particularly helpful for women in the post-menopausal age group. In a recent population-based Swedish study 893 women aged 60 to 75 performed VSS at home, with $4.4 \%$ positivity and $2.5 \%$ persistent positivity at 2 nd sampling (64). The authors concluded that vaginal self-sampling at home was well-accepted among women in this age group.

Although the present results did not show significantly increased comorbid conditions among the patients with detected recurrence, further inspection of Tables I-III suggests that in individual cases these disorders, especially autoimmune conditions, may have been contributory. Notably, in a long-term large-scale Australian study (65), autoimmune conditions were found to be associated with increased cervical dysplasia, supporting the need for 'expansion of cervical cancer preventative programs to include these at-risk females'.

A recent meta-analysis indicates that, compared to surgical excision alone, prophylactic HPV vaccination is associated with a significantly decreased risk of recurrence among patients treated for high-grade CIN (66). Unfortunately, data concerning HPV vaccination are not available for the present cohort, and would be an important consideration in future studies. Co-infection with Epstein-Barr virus, EBV, might also be considered in future studies, given some reports of its possible contribution to progression of cervical lesions (67).

In conclusion, the results of the present study indicate that four factors significantly and independently contribute to the risk of recurrent high-grade cervical intraepithelial neoplasia, CIN. Among these, incomplete excision of the CIN lesion was the most significant predictor of recurrence, and, therefore, warrants more intensive subsequent screening, regardless of the early post-conization HPV findings. An early post-conization positive HPV finding was also a powerful, independent predictor recurrent high-grade CIN. Nevertheless, over one-third of the patients with detected recurrence had a negative early post-conization HPV finding. These patients were returned to routine screening, at which time in most (but not all the cases), the HPV finding was positive. This underscores the vital need for repeated evaluation of HPV. Especially during this on-going pandemic crisis, vaginal self-sampling, VSS, for HPV is strongly recommended. However, caution and further investigation regarding VSS are still needed insofar as the cervical pathology is glandular and not squamous. Abnormal early post-conization cytology was also a significant independent predictor of recurrent high-grade CIN. This finding supports the current national guidelines of more intensive follow-up for patients with abnormal cytology post-conization.

Women aged 45 or above were also at higher risk of recurrence. Besides implementing all the above-recommended measures vis-à-vis the three other independent risk factors, special attention is warranted to women in this age group. Among the pertinent issues are choice of surgical modality (laser may be preferable), performance of wider, more complete excision, as well as the increased likelihood of an inadequate colposcopic examination associated with transition zone $3, \mathrm{TZ} 3$, which is often found among women in the more senior age group.

Although not statistically significant, relevant comorbidity, warrants consideration in clinical decision-making about follow-up. This appears to be especially relevant for autoimmune conditions.

Overall, women who have been treated for high-grade cervical intraepithelial neoplasia are at increased risk for recurrent disease and progression to cervical cancer, and therefore require careful, individualized follow-up to avoid these potential adverse consequences. The results of the present study provide insights that can substantially contribute to the practical realization of this aim.

\section{Acknowledgements}

Not applicable.

\section{Funding}

The present study was supported by the Swedish Cancer Foundation (grant no. 110544, CAN2011/471), the Karolinska Institute (Cancer Strategic Grants; grant no. 5888/05-722), the Swedish Research Council (grant no. 521-2008-2899), the Stockholm County Council (grant nos. 20130097 and 20160155) and the Gustaf V Jubilee Fund (grant nos. 154022 and 151202).

\section{Availability of data and materials}

The datasets used and/or analyzed during the current study are available from the corresponding author on reasonable request.

\section{Authors' contributions}

SAn, DM, SAl and EÖ confirm the authenticity of the raw data. SAn conceived and designed the study, performed colposcopy, cervical sampling and punch biopsies of visible lesions, assessed the authenticity of the raw data, reviewed the data, collected the related literature, and revised the manuscript. 
DM participated in the design and conception of the study, assessed the authenticity of the raw data, prepared the data set for analysis, collected related literature, participated in the statistical analysis, drafting and revision of the manuscript. KB performed the statistical analysis, collected the related literature, wrote and revised the manuscript. SAl participated in the design and conception of the study, assessed the authenticity of the raw data, prepared the data set for analysis, collected the related literature and revised the manuscript. EÖ participated in the design and conception of the study, was responsible for identifying and recruiting all the participants, met with all the participants, gave the instructions for self-sampling, assessed the authenticity of the raw data, prepared the data set for analysis, collected the related literature and revised the manuscript. MM participated in the design and conception of the study, performed colposcopy, cervical sampling and punch biopsies of visible lesions, and revised the manuscript. All authors read and approved the final manuscript.

\section{Ethics approval and consent to participate}

Karolinska Ethics Committee approved the study protocol (approval nos. 2006/1273-31, 2014/2034-3). All participants provided written informed consent.

\section{Patient consent for publication}

Not applicable.

\section{Competing interests}

The authors declare that they have no competing interests.

\section{References}

1. Bray F, Ferlay J, Soerjomataram I, Siegel R, Torre L and Jemal A: Global cancer statistics 2018: GLOBOCAN estimates of incidence and mortality worldwise for 36 cancers in 185 countries. CA Cancer J Clin 68: 394-424, 2018.

2. Hemminki K, Li X and Vaittinen P: Time trends in the incidence of cervical and other genital squamous cell carcinomas and adenocarcinomas in Sweden, 1958-1996. Eur J Obstet Gynecol Reprod Biol 101: 64-69, 2002.

3. Gunnell AS, Ylitalo N, Sandin S, Sparén P, Adami HO and Ripatti S: A longitudinal Swedish study on screening for squamous cell carcinoma and adenocarcinoma: Evidence of effectiveness and overtreatment. Cancer Epidemiol Biomarkers Prev 16: 2641-2648, 2007.

4. Screening SNC, Registry. Förebyggande av livmoderhalscancer i Sverige 2017. Available from: http://www.nkcx.se/templates/_ rsrapport_2017.pdf.

5. Andrae B, Kemetli L, Sparén L, Strander B, Ryder W, Dillner J and Törnberg S: Screening-preventable cervical cancer risks: Evidence from a nationwide audit in Sweden. J Natl Cancer Inst 100: 622-629, 2008.

6. Ebisch R, Rutten D, IntHout J, Melchers W, Massuger L, Bulten J, Bekkers R and Siebers A: Long-lasting increased risk of human papillomavirus-related carcinomas and premalignancies after cervical intraepithelial neoplasia grade 3: A population-based cohort study. J Clin Oncol 35: 2542-2550, 2017.

7. Pettersson BF, Hellman K, Vaziri R, Andersson $S$ and Hellström AC: Cervical cancer in the screening era: Who Fell victim in spite of successful screening programs? J Gynecol Oncol 22: 76-82, 2011.

8. Strander B, Andersson-Ellström A, Milsom I and Sparén P: Long term risk of invasive cancer after treatment for cervical intraepithelial neoplasia grade 3: Population based cohort study. BMJ 335: 1077, 2007.
9. Hoffman S, Le T, Lockhart A, Sanusi A, Dal Santo L, Davis M, McKinney D, Brown M, Poole C, Willame C and Smith JS: Patterns of persistent HPV infection after treatment for cervical intraepithelial neoplasia (CIN): A systematic review. Int J Cancer 141: 8-23, 2017.

10. Arbyn M, Ronco G, Anttila A, Meijer CJ, Poljak M, Ogilvie G, Koliopoulos G, Nauclen P, Sankaranarayanan R and Peto J: Evidence regarding human papillomavirus testing in secondary prevention of cervical cancer. Vaccine 30 (Suppl 5): F88-F99, 2012.

11. Brismar S, Johansson B, Borjesson M, Arbyn M and Andersson S: Follow-up after treatment of cervical intraepithelial neoplasia by human papillomavirus genotyping. Am J Obstet Gynecol 201: 17.e1-e8, 2009.

12. Kocken M, Uijterwaal M, de Vries A, Berkhof J, Ket J, Helmerhorst $\mathrm{J}$ and Meijer C: High-risk papillomavirus testing versus cytology in predicting post-treatment disease in women treated for high-grade cervical disease: A systematic review and meta-analysis. Gynecol Oncol 125: 500-507, 2012.

13. Persson M, Brismar Wendel S, Ljungblad L, Johansson B, Weiderpass E and Andersson S: High-risk human papillomavirus E6/E7 mRNA and L1 DNA as markers of residual/recurrent cervical intraepithelial neoplasia. Oncol Rep 28: 346-352, 2012.

14. Garutti P, Borghi C, Bedoni C, Bonaccorsi G, Greco P, Tognon M and Martini F: HPV-based strategy in follow-up of patients treated for high-grade cervical intra-epithelial neoplasia: 5-year results in a public health surveillance setting. Eur J Obstet Gynecol Reprod Biol 210: 236-241, 2017.

15. Bruhn L, Andersen S and Hariri J: HPV-testing versus HPV-cytology co-testing to predict the outcome after conization. Acta Obstet Gynecol Scand 97: 758-765, 2018.

16. Hermansson RS, Olovsson M, Hoxell E and Lindström AK: HPV prevalence and HPV-related dysplasia in elderly women. PLoS One 13: e0189300, 2018.

17. Rodriguez A, Schiffman M, Herrero R, Hildesheim A, Bratti C, Sherman M, Solomon D, Guillén D, Alfaro M, Morales J, et al: Longitudinal study of human papillomavirus persistence and cervical intraepithelial neoplasia grade 2/3: Critical role of duration of infection. J Natl Cancer Inst 102: 315-324, 2010.

18. Plummer M, Schiffman M, Castle P, Maucort-Boulch D and Wheeler C: A 2-year prospective study of human papillomavirus persistence among women with a cytological diagnosis of atypical squamous cells of undetermined significance or low-grade squamous intraepithelial lesion. J Infect Dis 195: 1582-1589, 2007.

19. Alder S, Megyessi D, Sundström K, Östensson E, Mints M, Belkić K, Arbyn M and Andersson S: Incomplete excision of cervical intraepithelial neoplasia as a predictor of the risk of recurrent disease-a 16 year follow-up study. Am J Obstet Gynecol 222: 172e1-172e12, 2020.

20. Arbyn M, Redman C, Verdoodt F, Kyrgiou M, Tzafetas M, Ghaem-Maghami S, Petry KU, Leeson S, Bergeron C, Nieminen $\mathrm{P}$, et al: Incomplete excision of cervical precancer as a predictor of treatment failure: A systematic review and meta-analysis. Lancet Oncol 18: 1665-1679, 2017.

21. Fernández-Montolí ME, Tous S, Medina G, Castellarnau M, García-Tejedor A and de Sanjosé S: Long-term predictors of residual or recurrent cervical intraepithelial neoplasia 2-3 after treatment with a large loop excision of the transformation zone: A retrospective study. BJOG 127: 377-387, 2020.

22. Reich O, Pickel H, Lahousen M, Tamussino K and Winter R: Cervical intraepithelial neoplasia III: long-term outcome after cold-knife conization with clear margins. Obstet Gynecol 97: 428-430, 2001.

23. Reich O, Lahousen M, Pickel H, Tamussino K and Winter R: Cervical intraepithelial neoplasia III: Long-term follow-up after cold-knife conization with involved margins. Obstet Gynecol 99: 193-196, 2002.

24. Ghaem-Maghami S, Sagi S, Majeed G and Soutter WP: Incomplete excision of cervical intraepithelial neoplasia and risk of treatment failure: A meta-analysis. Lancet Oncol 8: 985-993, 2007.

25. Östensson E, Belkić K, Ramqvist T, Mints M and Andersson S: Self-sampling for high-risk human papillomavirus as a follow-up alternative after treatment of high-grade cervical intraepithelial neoplasia. Oncol Lett 21: 240, 2021.

26. Alder S: Prevention of Cervical Cancer in Countries with High and Low Incidence of the Disease. Doctoral Dissertation Stockholm, Sweden: Karolinska Institute, 2018.

27. Andersson S,Belkić K, Mints M and Östensson E: Is self-sampling to test for HPV an acceptable option among women who have been treated for high-grade cervical intraepithelial neoplasia? PLoS One 13: e0199038, 2018. 
28. Andersson S, Belkić K, Safer Demirbüker S, Mints M and Östensson E: Perceived cervical cancer risk among women treated for high-grade cervical intraepithelial neoplasia: The importance of specific knowledge. PLoS One 12: e190156, 2017.

29. Richart RM: Cervical intraepithelial neoplasia. Pathol Annu 8: 301-328, 1973 .

30. Jordan J, Martin-Hirsch P, Arbyn M, Schenck U, Baldauf JJ, Da Silva D, Anttila A, Nieminen P and Prendiville W: European guidelines for clinical management of abnormal cervical cytology-part-2. Cytopathology 20: 5-16, 2009.

31. Solomon D, Davey D, Kurman R, Moriarty A, O'Connor D, Prey M, Raab S, Sherman M, Wilbur D, Wright T, et al: The 2001 Bethesda System: Terminology for reporting results of cervical cytology. JAMA 287: 2114-2119, 2002.

32. Martin-Hirsch PL, Paraskevaidis E, Bryant A, Dickinson HO and Keep SL: Surgery for cervical intraepithelial neoplasia. Cochrane Database Syst Rev: CD001318, 2010 doi: 10.1002/14651858. CD001318.pub2.

33. Ghaem-Maghami S, De-Silva D, Tipples M, Lam S, Perryman K and Soutter W: Determinants of success in treating cervical intraepithelial neoplasia. BJOG 118: 679-684, 2011.

34. Fallani MG, Penna C, Fambrini $M$ and Marchionni M: Laser $\mathrm{CO}_{2}$ vaporization for high-grade cervical intraepithelial neoplasia: A long-term follow-up series. Gynecol Oncol 91: 130-133, 2003.

35. Wongtiraporn W, Laiwejpithaya S, Sangkarat S, Benjapibal M, Rattanachaiyanont M, Ruengkhachorn I, Chaopotong P and Laiwejpithaya S: Long term outcomes of laser conization for high grade cervical intraepithelial neoplasia in Thai women. Asian Pac J Cancer Prev 15: 7757-7761, 2014.

36. Frank J: The colposcopic examination. J Midwifery Womens Health 53: 447-452, 2008

37. Manley K, Simms R, Platt S, Patel A and Bahl R: Unsatisfactory colposcopy: Clinical decision making in conditions of uncertainty. BMC Med Inform Decis Mak 17: 125, 2017.

38. Baasland I, Hagen B, Vogt C, Valla M and Romundstad PR Colposcopy and additive diagnostic value of biopsies from colposcopy-negative areas to detect cervical dysplasia. Acta Obstet Gynecol Scand 95: 1258-1263, 2016.

39. Bogani G, Pinelli C, Chiappa V, Martinelli F, Lopez S, Ditto A and Rapagliesi F: Age-specific predictors of cervical dysplasia recurrence after primary conization: Analysis of 3,212 women. J Gynecol Oncol 31: e60, 2020

40. Andersson S, Larson B, Hjerpe A, Silfverswärd C, Sällström J, Wilander E and Rylander E: Adenocarcinoma of the uterine cervix: The presence of human papillomavirus and the method of detection. Acta Obstet Gynecol Scand 82: 960-965, 2003.

41. Dahlström L, Ylitalo N, Sundström K, Palmgren J, Ploner A, Eloranta S, Sanjeevi C, Andersson S, Rohan T, Dillner J, et al: Prospective study of human papillomavirus and risk of cervical adenocarcinoma. Int J Cancer 127: 1923-1930, 2010

42. Dillner J: Covid-19: Challenges and opportunities for cancer screening: An example from cervical cancer in Sweden. International Agency for Research on Cancer COVID-19 and cancer screening. May, 2021.

43. de Pelsemaeker MC, Guiot Y, Vanderveken J, Galant C and Van Bockstal M: The impact of the COVID-19 pandemic and the associated Belgian governmental measures on cancer screening, surgical pathology and cytopathology. Pathobiology 88: 46-55, 2021.

44. Feletto E, Grogan P, Nickson C, Smith M and Canfell K: How has COVID-19 impacted cancer screening? Adaptation of services and the future outlook in Australia. Public Health Res Pract 30 e3042026, 2020

45. Kregting LM, Kaljouw S, de Jonge L, Jansen EEL, Peterse EFP, Heijnsdijk EAM, van Ravesteyn NT, Lansdorp-Vogelaar I and de Kok IMCM: Effects of cancer screening restart strategies after COVID-19 disruption. Br J Cancer 12: 1516-1523, 2021

46. Miller M, Xu L, Qin J, Hahn E, Ngo-Metzger Q, Mittman B, Tewari D, Hodeib M, Wride P, Saraiya M and Chao CR: Impact of COVID-19 on cervical cancer screening rates among women aged 21-65 in a large integrated health care system-Southern California January 1-September 30, 2019 and January 1-September 30, 2020 MMWR Morb Mortal Wkly Rep 70: 109-113, 2021.

47. Gorin SNS, Jimbo M, Heizelman R, Harmes K and Harper D: The future of cancer screening after COVID-19 may be at home. Cancer 127: 498-503, 2021.

48. Burger E, Jansen E, Killen J, de Kok U, Smith M, Sy S, Dunnewind N, Campos N, Haas J, Kobrin S, et al: Impact of COVID-19-related care disruptions on cervical cancer screening in the United States. J Med Screen 28: 213-216, 2021.
49. Nogami Y, Kobayashi Y, Tsuji K, Yokota M, Nishio H, Nakamura M, Yamagami W, Morisada T, Tominaga E, Banno K and Aoki D: Impact of the COVID-19 epidemic at a high-volume facility in gynecological oncology in Tokyo, Japan: A single-center experience. J Ovarian Res 13: 105, 2020.

50. Ajenifuja K, Belinson J, Goldstein A, Desai K, de Sanjose S and Schiffman M: Designing low-cost, accurate cervical screening strategies that take into account COVID-19: A role for self-sampled HPV typing. Infect Agent Cancer 15: 61, 2020.

51. Ciavattini A, Carpini G, Giannella L, Arbyn M, Kyrgiou M, Joura E, Sehouli J,Carcopino X,Redman C,Nieminen P, et al:European Federation for Colposcopy (EFC) and European Society of Gynaecological Oncology (ESGO) joint considerations about human papillomavirus (HPV) vaccination, screening programs, colposcopy, and surgery during and after the COVID-19 pandemic. Int J Gynecol Cancer 30: 1097-1100, 2020.

52. Cohen M, Powell A, Coleman J, Keller J, Livingston A and Anderson J: Special ambulatory gynecologic considerations in the era of coronavirus disease 2019 (COVID-19) and implications for future practice. Am J Obstet Gynecol 223: 372-378, 2020.

53. Feldman S and Haas J: How the corona disease-2019 may improve care: Rethinking cervical cancer prevention. J Natl Cancer Inst 113: djaa089, 2020.

54. Arbyn M, Verdoodt F, Snijders P, Verhoef V, Suonio E, Dillner L, Minozzi S, Bellisario C,Banzi R,ZhaoFH, et al: Accuracy of human papillomavirus testing on self-collected versus clinician-collected samples: A meta-analysis. Lancet Oncol 15: 172-183, 2014.

55. Arbyn M, Snijders PJ, Meijer CJ, Berkhof J, Cuschieri K, Kocjan BJ and Poljak M: Which high-risk HPV assays fulfill criteria for use in primary cervical cancer screening? Clin Microbiol Infect 21: 817-826, 2015.

56. Jentschke M, Chen K, Arbyn M, Hertel B, Noskowicz M, Soergel P and Hillemans P: Direct comparison of two vaginal self-sampling devices for the detection of human papillomavirus infections. J Clin Virol 82: 46-50, 2016.

57. Bergengren L, Kaliff M, Larsson G, Karlsson M and Helenius G: Comparison between professional sampling and self-sampling for HPV-based cervical cancer screening among postmenopausal women. Int J Gynecol Obstet 142: 359-364, 2018

58. Östensson E, Hellström AC, Hellman K, Gustavsson I, Gyllensten U, Wilander E, Zethraeus N and Andersson S: Projected cost-effectiveness of repeat high-risk human papillomavirus testing using self-collected vaginal samples in the Swedish cervical cancer screening program. Acta Obstet Gynecol Scand 92: 830-840, 2013.

59. Galbraith KV, Gilkey MB, Smith JS, Richman AR, Barclay L and Brewer NT: Perceptions of mailed HPV self-testing among women at higher risk for cervical cancer. J Community Health 39: 849-856, 2014.

60. Arrossi S, Ramos S, Straw C, Thouyaret L and Orellana L: HPV testing: A mixed-method approach to understand why women prefer self-collection in a middle-income country. BMC Public Health 16: 832, 2016.

61. Racey C and Gesink D: Barriers and facilitators to cervical cancer screening among women in rural Ontario, Canada: The role of self-collected HPV testing. J Rural Health 32: 136-145, 2016.

62. Mao C, Kulasingam S, Whitham H, Hawes S, Lin J and Kiviat N: Clinician and patient acceptability of self-collected human papillomavirus testing for cervical cancer screening. J Womens Health 26: 609-615, 2017.

63. Andersson S, Belkić K, Mints M and Östensson E: Acceptance of self-sampling among long-term cervical screening non-attenders with HPV positive results: Promising opportunity for specific cancer education. J Cancer Educ 36: 126-133, 2021.

64. Lindström AK, Hermansson RS, Gustavsson I, Hedlund Lindberg J, Gyllensten U and Olovsson M: Cervical dysplasia in elderly women performing repeated self-sampling for HPV testing. PLoS One 13: e0207714, 2018.

65. Foster E, Malloy M, Jokubaitis V, Wrede D, Butzkueven H, Sasadeusz J, Van Doornum S, Macrae F, Unglik G, Brotherton J and van der Walt A: Increased risk of cervical dysplasia in females with autoimmune conditions-results from an Australia database linkage study. PLoS One 15: e0234813, 2020.

66. Jentschke M,Kampers J, Becker J, Sibbertsen P and Hillemanns P: Prophylactic HPV vaccination after conization: A systematic review and meta-analysis. Vaccine 38: 6402-6409, 2020.

67. Joharinia N, Faghihinezhad S, Seyedi K, Farhadi A, Hosseini SY, Safaei A, Baharampour H and Sarvari J: Co-existing of HSV1/2 or EBV infection with the presence of high-risk HPV DNA in cervical lesions in the southwest of Iran. Asian Pac J Cancer Prev 21: 1459-1464, 2020.

This work is licensed under a Creative Commons Attribution-NonCommercial-NoDerivatives 4.0 International (CC BY-NC-ND 4.0) License. 